\title{
O PROGRAMA DE EDUCAÇÃO TUTORIAL E A FORMAÇÃO DE ENGENHEIROS CIVIS PREPARADOS PARA O FUTURO MERCADO DE TRABALHO
}

\author{
DOI: 10.37702/2175-957X.COBENGE.2021.3528
}

Leonardo Meiguel - meiguelleonardo@gmail.com

Universidade Federal do Paraná

Washington Luiz 66

17510-406 - Marília - SP

Cristiane Santana Monteiro dos Santos - cristianemonteiro@ufpr.br

UNIVERSIDADE FEDERAL DO PARANÁ

Rua Vicente de Carvalho 450

82940-370 - Curitiba - PR

Elvidio Gavassoni Neto - gavassoni@ufpr.br

Universidade Federal do Paraná

Rua Gabriela Mistral 149

80540-015 - Curitiba - PR

João Antonio Comine da Silva - jantoniocominedasilva@gmail.com Universidade Federal do Paraná

Rua José Manuel Voluz 1540

81900-043 - Curitiba - PR

Guilherme Conde Dias - guilherme.conde.dias@gmail.com

Universidade Federal do Paraná

marechal anor teixeira dos santos 557

82650-120 - Curitiba - PR

Resumo: A quarta revolução industrial trouxe à sociedade a necessidade de compreensão das novas tecnologias, o aperfeiçoamento de habilidades não técnicas e suas aplicações no mercado de trabalho. Os engenheiros civis entram nesse perfil de profissional, pois trabalham com a resolução de problemas práticos complexos, seus trabalhos exigem habilidades sociocomportamentais e a utilização de novas tecnologias visando a produtividade e consciência ambiental. A 
Universidade Federal do Paraná, a mais de 37 anos possui o Programa de Educação Tutorial (PET) em Engenharia Civil, o qual é um programa de educação pioneiro instituído pelo MEC que busca integrar ações inovadoras articulando a tríade universitária de ensino, pesquisa e extensão. O PET nessa articulação desenvolve atividades exercendo trabalho colaborativo, horizontalidade, desenvolve a organização interna e realiza planejamentos, tudo isso sob orientação de um professor tutor. Com a finalidade de analisar quanto as atividades realizadas pelo grupo PET colabora na formação de engenheiros civis, foi realizado um estudo das atividades do grupo no período 2013-2021 tendo como base um trabalho dos países da União Europeia que lista 59 habilidades relevantes aos engenheiros civis. Constata-se que o grupo em suas atividades exercitou $85 \%$ das habilidades mencionadas, confirmando a importância do caráter do programa de educação tutorial na formação de engenheiros civis.

Palavras-chave: Indústria 4.0, construção civil, habilidades futuras. 


\section{O PROGRAMA DE EDUCAÇÃO TUTORIAL E A FORMAÇÃO DE ENGENHEIROS CIVIS PREPARADOS PARA O FUTURO MERCADO DE TRABALHO}

\section{INTRODUÇÃO.}

A quarta revolução industrial trouxe à humanidade uma grande diversidade de desafios. É necessário compreender a amplitude dessa nova revolução, além de conhecer e utilizar suas ferramentas, como por exemplo a inteligência artificial (IA), a internet das coisas (IoT), a impressão 3D e o armazenamento de energia (SCHWAB, 2016). Esse avanço tecnológico cria a necessidade de uma maior qualificação de profissionais em inúmeras áreas de conhecimento, seja por qualificação em habilidades técnicas e nãotécnicas. Sendo assim, a educação, em especial a de ensino superior, assume papel fundamental na formação profissional para a sociedade, devendo buscar produtividade e competitividade (SILVA; OLAVE, 2020).

Em um futuro próximo, o mercado de trabalho irá exigir o pleno conhecimento acerca dessas novas tecnologias, além disso também será importante o domínio das habilidades sociocomportamentais para que os profissionais possam desenvolver suas atividades em alta performance (AKYAZI et al., 2020). Porém, parte das Instituições de Ensino Superior (IES) possui baixo investimento em tecnologia avançada e necessitam trabalhar mais habilidades pessoais, multidisciplinares e práticas (SILVA; OLAVE, 2020).

Os engenheiros civis não são exceção à essas mudanças no mercado de trabalho. Engenheiros civis precisam lidar com a resolução de problemas práticos complexos, cujos dados são incompletos e cujas demandas (muitas vezes conflitantes) são diversas (CAMPUS; PENRITH, 2003). Conciliar demandas de clientes, de agências reguladoras, de organismos ambientais e governamentais demandam uma série de habilidades não técnicas quase sempre não praticadas por metodologias de ensino tradicionais centradas na figura dos docentes com aulas expositivas (FELDER; BRENT, 2004a).

Outro fator desafiador é que grande parte das habilidades técnicas trabalhadas nos currículos de graduação em engenharia civil são, no mercado de trabalho, desempenhadas por computadores e não engenheiros (APARICIO et al., 2007). Uma vez liberados de análises matemáticas e cálculos extensivos, os engenheiros civis possuem mais tempo para exercer habilidades não-técnicas importantes como pensamento crítico e criatividade (NAVON, 2020). Assim os engenheiros civis são demandados, em um nível cada vez maior, por habilidades técnicas e não-técnicas que o tempo passado na graduação não Ihes fornecem oportunidades de desenvolvimento (FELDER; BRENT, 2004b).

$\mathrm{Na}$ Universidade Federal do Paraná (UFPR), nos últimos anos, houveram discussões e estudos sobre a remodelagem do projeto pedagógico no curso de engenharia civil (PESQUISA; SILVA, J. A. C.; PRADO, C. R.; GONÇALVES, L. W.; SIMIONATO, N. M.; OLIVEIRA, N. C.; GAVASSONI, 2019), tendo em vista o avanço tecnológico (TIBONI; PEREIRA, 2019). Todavia, dentro do atual contexto da graduação de Engenharia Civil existe ainda grande espaço para o afrontamento desse contexto de demanda por novas habilidades profissionais dos futuros engenheiros civis em formação na instituição. Nesse contexto, marcado pela necessidade de existência de oportunidades eficientes para trabalhar tais habilidades por meio de atividades extracurriculares, pode-se citar 0 Programa de Educação Tutorial (PET) como uma importante ferramenta para atender tais necessidades. O PET é um programa que visa proporcionar uma formação ampla e de qualidade a alunos de graduação das Instituições de Ensino Superior, articulando a tríade universitária: ensino, pesquisa e extensão (MARTIN, 2005). 
O PET Engenharia Civil UFPR possui mais de 37 anos de história (2008, PET 25 ANOS) e possui como missão promover a melhoria do curso de Engenharia Civil articulando ações inovadoras de ensino, pesquisa e extensão, de forma técnica e humana. $O$ trabalho do grupo se baseia na horizontalidade e no trabalho colaborativo, ou seja, não há níveis de hierarquia e todos possuem o mesmo poder de decisão. Sendo assim, nota-se que seus membros desenvolvem habilidades importantes, como respeito à diversidade, a empatia, resiliência diante situações de tensão e criatividade, além do comprometimento e lealdade aos colegas do grupo e aos princípios do PET (MARTIN, 2005). Além disso, para realização dessas atividades o grupo realiza planejamentos anuais, possui uma organização interna com departamentos e coordenadorias, e ainda nos projetos que desenvolve realiza a estruturação com gerente e grupo de trabalho (PRIMO et al., 2019).

Tanto a estruturação interna do grupo PET Engenharia Civil quanto as atividades que vem planejando e executando ao longo de sua história atendem às necessidades de desenvolvimento de habilidades técnicas e não técnicas de engenheiros civis nas condições atuais e futuras do mercado de trabalho. O objetivo deste estudo é avaliar a extensão da experiência de desenvolvimento profissional, adequado às inovações no mercado de trabalho, atuais e futuras, provida aos membros do grupo PET Engenharia Civil pelas suas atividades. Para isso utilizam-se as 45 habilidades gerais e 14 habilidades específicas necessárias aos engenheiros civis recentemente listadas pelo trabalho de Akyazi e coautores (2020) como tendências futuras em face das exigências do mercado de trabalho dos países da União Europeia.

Utilizando-se os relatórios de planejamento e de atividades do grupo PET Engenharia Civil da UFPR no período de 2013 a 2021 identificou-se as realizações do grupo nesse período e quais dentre as habilidades da lista de Akyazi e coautores (2020) foram trabalhadas por cada uma das atividades do grupo ao longo de 9 anos. Os resultados foram então analisados mostrando o número de habilidades exercidas por ano, as habilidades mais trabalhadas e aquelas que não foram contempladas pelas atividades do grupo PET Engenharia Civil. Esse trabalho constitui-se em uma importante ferramenta no planejamento do grupo para os anos vindouros e também avalia o potencial de formação ampla fornecido pelo programa de educação tutorial para engenheiros civis preparados em termos de habilidades para as demandas futuras do mercado de trabalho.

\section{METODOLOGIA}

Nesta seção são apresentados os métodos empregados para o estudo. Primeiramente são apresentadas as 59 habilidades listadas pelo trabalho de Akyazi e coautores (2020) utilizado como base para este estudo. Em seguida as atividades realizadas pelo grupo PET Engenharia Civil no período 2013-2021 são listadas.

Utiliza-se a mesma nomenclatura encontrada na referência base deste estudo (AKYAZI et al., 2020) em que as habilidades profissionais requeridas pelo mercado de trabalho futuro são classificadas em habilidades gerais e habilidades específicas. Apenas as competências referentes ao perfil 4 do trabalho de Akyazi e coautores (2020) foram utilizadas, uma vez que esse é o perfil referente aos engenheiros civis.

No Quadro 1 são apresentadas as 45 habilidades gerais, enquanto no Quadro 2 são listadas as 14 habilidades específicas segundo Akyazi e coautores (2020). Utiliza-se, com objetivo de simplificação da apresentação e da discussão dos resultados a nomenclatura simplificada de referência (Ref.) dada por HXX, onde XX é a ordem numérica de apresentação das habilidades mostradas nos Quadros 1 e 2 variando entre 1 e 59. Manteve-se a mesma ordem sequencial das habilidades encontradas no trabalho de referência (AKYAZI et al., 2020). 
Quadro 1 - Habilidades gerais

\begin{tabular}{|c|c|c|c|}
\hline Ref. & Habilidade & Ref. & Habilidade \\
\hline $\mathrm{H} 1$ & Inspeção e monitoramento & $\mathrm{H} 24$ & Experiência pessoal \\
\hline $\mathrm{H} 2$ & Habilidades digitais básicas & $\mathrm{H} 25$ & Etica profissional \\
\hline $\mathrm{H} 3$ & Análise de dados avançada & $\mathrm{H} 26$ & Empatia cultural \\
\hline $\mathrm{H} 4$ & Habilidades matemáticas & $\mathrm{H} 27$ & Autonomia no trabalho \\
\hline $\mathrm{H} 5$ & Cibersegurança & $\mathrm{H} 28$ & Escuta ativa \\
\hline $\mathrm{H} 6$ & Uso de ferramentas de comunicação digital complexas & $\mathrm{H} 29$ & Numeracia básica e comunicação \\
\hline $\mathrm{H} 7$ & Habilidades avançadas de TI e programação & $\mathrm{H} 30$ & Entrada e processamento de dados básicos \\
\hline $\mathrm{H} 8$ & IOT & H31 & Literacia avançada \\
\hline $\mathrm{H} 9$ & Big Data & H32 & Análise quantitativas e estatísticas \\
\hline $\mathrm{H} 10$ & Inteligência Artificial (IA) & H33 & Processamento de informações complexas \\
\hline $\mathrm{H} 11$ & Tecnologia de sensores & H34 & Habilidades linguísticas adequadas \\
\hline $\mathrm{H} 12$ & Realidade Aumentada (AR) & $\mathrm{H} 35$ & Análise de processo \\
\hline $\mathrm{H} 13$ & Aprendizado automático (de máquina) & H36 & Criatividade \\
\hline $\mathrm{H} 14$ & Business Intelligence & H37 & Resolução de problemas complexos \\
\hline $\mathrm{H} 15$ & Gestão de Segurança da Informação & H38 & Resolução de conflitos \\
\hline $\mathrm{H} 16$ & Comunicação avançada & H39 & Gerenciamento de problemas \\
\hline $\mathrm{H} 17$ & Relacionamento interpessoal e empatia & $\mathrm{H} 40$ & Gerenciamento de riscos \\
\hline $\mathrm{H} 18$ & Liderança e gerenciamento de equipes & $\mathrm{H} 41$ & Consciência ambiental \\
\hline $\mathrm{H} 19$ & Empreendedorismo e proatividade & $\mathrm{H} 42$ & Eficiência energética \\
\hline $\mathrm{H} 2 \mathrm{O}$ & Flexibilidade e adaptação às mudanças & $\mathrm{H} 43$ & Reutilização / reciclagem de recursos \\
\hline $\mathrm{H} 21$ & Aprendizado contínuo & $\mathrm{H} 44$ & Gestão de resíduos (redução e reutilização) \\
\hline $\mathrm{H} 22$ & Ensino e tutoria & $\mathrm{H} 45$ & Avaliação do ciclo de vida \\
\hline $\mathrm{H} 23$ & Pensamento crítico e tomada de decisões & & \\
\hline
\end{tabular}

Fonte: (AKYAZI et al., 2020)

Quadro 2 - Habilidades específicas

\begin{tabular}{|c|c|}
\hline Ref. & Habilidade \\
\hline H46 & Aplicação de novas tecnologias à engenharia civil \\
\hline H47 & Drones \\
\hline H48 & Metodologia BIM \\
\hline H49 & Construção robótica \\
\hline H50 & Impressão 3D \\
\hline H51 & Digitalização a laser 3D \\
\hline H52 & Novos materiais de construção \\
\hline H53 & Procedimentos de qualidade relacionados à transformação digital \\
\hline H54 & Eficiência energética de edifícios e infraestruturas \\
\hline H55 & Gestão de riscos relacionados às mudanças climáticas \\
\hline H56 & Gestão sustentável de resíduos - economia circular \\
\hline H57 & Gestão de recursos sustentáveis \\
\hline H58 & Gerenciamento de Projetos \\
\hline H59 & Ergonomia \\
\hline
\end{tabular}

Fonte: (AKYAZI et al., 2020)

Em seguida realizou-se a análise dos planejamentos anuais (UFPR, 2013a, 2014a, 2015a, 2016a, 2017a, 2018a, 2019a, 2020a, 2021) e relatórios anuais (UFPR, 2013b, 2014b, 2015b, 2016b, 2017b, 2018b, 2019b, 2020b) de atividades do grupo PET encaminhados ao Ministério da Educação. Nesses documentos é possível encontrar a descrição, os objetivos, a metodologia, os resultados e as conclusões de cada um dos 51 tipos de atividades planejadas e realizadas entre os anos de $2013-2021$. Para o ano de 2021, por se tratar do ano corrente, foi utilizado apenas o relatório de planejamento.

Os tipos de atividades encontrados nos relatórios e planejamentos são listados no Quadro 3, onde também são apresentados os períodos de ocorrência para cada um dos tipos de atividades. Com o intuito de simplificação da apresentação e da discussão dos 
resultados do estudo utiliza-se a nomenclatura simplificada de referência (Ref.) dada por AXX, onde XX é a ordem numérica de apresentação das atividades realizadas pelo grupo PET Engenharia Civil mostradas no Quadro 3 variando entre 1 e 51 atividades

Quadro 3 - Atividades realizadas no período de 2013-2021.

\begin{tabular}{|c|c|c|c|}
\hline Ref. & Atividade & Ref. & Atividade \\
\hline A1 & Articulação Ensino-Extensão & A27 & Organização Eventos Técnico-Científicos \\
\hline A2 & Atividades de extensão organização do XXV ENAPET & A28 & Palestras Técnicas \\
\hline A3 & Atividades ligadas à Estrutura Interna do Grupo & A29 & Parceria Passeio Público \\
\hline A4 & BIM & A30 & Parceria Prefeitura da Cidade Universitária \\
\hline A5 & Café com Engenheiro & A31 & Pavimentos Permeáveis \\
\hline A6 & Capacitação de Mão de Obra & A32 & PBL - Estruturas de Madeira \\
\hline A7 & Ciclo de Seminários & A33 & PBL - Introdução à Engenharia \\
\hline A8 & Circuito Arquitetura e Engenharia Civil & A34 & PBL - Sistemas de Transportes \\
\hline A9 & Circuito de Empreendedorismo & A35 & Pesquisa Científica RUMO \\
\hline A10 & Competição de Pontes de Papel & A36 & Pesquisa e Escrita Científica \\
\hline A11 & Cursos, Palestras e Visitas Técnicas & A37 & PET 35 ANOS \\
\hline A12 & Desafio Civil & A38 & PET em Campo \\
\hline A13 & Desafio de Taludes & A39 & PET por um dia \\
\hline A14 & Desafio Intermodal Engenharia de Tráfego & A40 & PET Talks \\
\hline A15 & Discussões sobre Educação & A41 & PETCast \\
\hline A16 & Engenharia para Leigos & A42 & Projeto Copa \\
\hline A17 & Eventos Internos & A43 & Repensando Civil \\
\hline A18 & Feira de Cursos de Profissões & A44 & Semana do Calouro \\
\hline A19 & Feira de Cursos do Colégio Estadual do Paraná & A45 & Semana do PET Civil \\
\hline A20 & Festival do Minuto & A46 & Talk Show \\
\hline A21 & Gravação Campanha Divulgação Kit Mola & A47 & Telhado Verde \\
\hline A22 & InterPET nas Escolas & A48 & Treinamento com o Tutor \\
\hline A23 & Jornal do PET Civil & A49 & Trunfo de Transportes \\
\hline A24 & Mitos \& Verdades Sobre o Trânsito & A50 & Valoriza Civil \\
\hline A25 & Mola Structural Kit & A51 & Vazios Urbanos \\
\hline A26 & Núcleo Docente Estruturante & & \\
\hline & & & \\
\hline
\end{tabular}

Fonte: Os autores

A análise das informações detalhadas dos documentos de relatórios e planejamentos anuais de atividades serviu como ferramenta de identificação das habilidades (Quadros 1 e 2) articuladas pelos tipos de atividades listadas no Quadro 3. Os resultados obtidos nessa análise são mostrados e discutidos na seção seguinte.

\section{RESULTADOS}

A aplicação da metodologia apresentada faz com que seja possível a obtenção dos resultados apresentados em 2 quadros (Quadros 4 e 5).

Apresenta-se no Quadro 4 a quantidade de vezes que uma determinada habilidade Hi encontrou oportunidade de ser desenvolvida pelo conjunto de atividades exercidas pelo grupo em um determinado ano. Nas linhas dos Quadros 4 estão as 59 habilidades descritas nos Quadros 1 e 2, já as colunas do Quadro 4 apresentam os 9 anos do período investigado por este trabalho. A última coluna apresenta o total de oportunidades ofertada pelo conjunto de atividades desenvolvidas pelo grupo em todo o período de 9 anos para 0 desenvolvimento de uma determinada habilidade Hi. A última linha representa a contagem do número de habilidades distintas trabalhadas em cada um dos anos do período em que foi realizado o estudo. 
Quadro 4 - Quantidade de habilidades por ano2013-2021.

\begin{tabular}{|c|c|c|c|c|c|c|c|c|c|c|}
\hline & 2013 & 2014 & 2015 & 2016 & 2017 & 2018 & 2019 & 2020 & 2021 & Total \\
\hline $\mathrm{H} 1$ & 5 & 4 & 6 & 7 & 7 & 11 & 15 & 4 & 7 & 66 \\
\hline $\mathrm{H} 2$ & 9 & 10 & 6 & 13 & 8 & 11 & 14 & 5 & 11 & 87 \\
\hline $\mathrm{H} 3$ & 5 & 5 & 0 & 7 & 4 & 5 & 6 & 4 & 6 & 42 \\
\hline $\mathrm{H} 4$ & 6 & 5 & 1 & 10 & 6 & 3 & 6 & 4 & 6 & 47 \\
\hline $\mathrm{H} 5$ & 0 & 0 & 0 & 0 & 0 & 0 & 0 & 0 & 1 & 1 \\
\hline $\mathrm{H} 6$ & 2 & 1 & 0 & 3 & 1 & 4 & 3 & 3 & 5 & 22 \\
\hline $\mathrm{H} 7$ & 0 & 0 & 0 & 2 & 1 & 2 & 2 & 2 & 2 & 11 \\
\hline $\mathrm{H} 8$ & 0 & 0 & 0 & 0 & 0 & 0 & 0 & 0 & 0 & 0 \\
\hline $\mathrm{H} 9$ & 0 & 0 & 0 & 0 & 0 & 0 & 0 & 0 & 1 & 1 \\
\hline $\mathrm{H} 10$ & 0 & 0 & 0 & 0 & 0 & 0 & 0 & 0 & 0 & 0 \\
\hline $\mathrm{H} 11$ & 2 & 0 & 0 & 0 & 0 & 0 & 0 & 0 & 0 & 2 \\
\hline $\mathrm{H} 12$ & 0 & 0 & 0 & 0 & 0 & 0 & 0 & 0 & 0 & 0 \\
\hline $\mathrm{H} 13$ & 0 & 0 & 0 & 0 & 0 & 0 & 0 & 0 & 0 & 0 \\
\hline $\mathrm{H} 14$ & 0 & 0 & 9 & 1 & 0 & 1 & 1 & 1 & 0 & 13 \\
\hline $\mathrm{H} 15$ & 1 & 1 & 9 & 2 & 1 & 2 & 2 & 1 & 2 & 21 \\
\hline $\mathrm{H} 16$ & 10 & 12 & 9 & 19 & 11 & 12 & 16 & 5 & 11 & 105 \\
\hline $\mathrm{H} 17$ & 10 & 12 & 6 & 19 & 11 & 12 & 16 & 5 & 11 & 102 \\
\hline $\mathrm{H} 18$ & 9 & 11 & 4 & 19 & 10 & 12 & 16 & 5 & 11 & 97 \\
\hline $\mathrm{H} 19$ & 4 & 9 & 9 & 10 & 6 & 4 & 4 & 3 & 8 & 57 \\
\hline $\mathrm{H} 20$ & 5 & 4 & 9 & 7 & 7 & 8 & 10 & 4 & 6 & 60 \\
\hline $\mathrm{H} 21$ & 10 & 12 & 9 & 19 & 11 & 11 & 15 & 5 & 11 & 103 \\
\hline $\mathrm{H} 22$ & 9 & 11 & 6 & 14 & 10 & 11 & 14 & 5 & 9 & 89 \\
\hline $\mathrm{H} 23$ & 10 & 12 & 9 & 19 & 11 & 10 & 14 & 5 & 11 & 101 \\
\hline $\mathrm{H} 24$ & 6 & 10 & 2 & 11 & 7 & 11 & 12 & 4 & 5 & 68 \\
\hline $\mathrm{H} 25$ & 10 & 12 & 9 & 19 & 11 & 12 & 16 & 5 & 11 & 105 \\
\hline $\mathrm{H} 26$ & 5 & 7 & 9 & 7 & 4 & 5 & 5 & 2 & 9 & 53 \\
\hline $\mathrm{H} 27$ & 10 & 12 & 9 & 19 & 11 & 12 & 16 & 5 & 11 & 105 \\
\hline $\mathrm{H} 28$ & 10 & 12 & 6 & 19 & 11 & 10 & 16 & 5 & 11 & 100 \\
\hline $\mathrm{H} 29$ & 10 & 12 & 5 & 19 & 11 & 12 & 16 & 5 & 10 & 100 \\
\hline $\mathrm{H} 30$ & 6 & 5 & 4 & 10 & 6 & 7 & 10 & 5 & 7 & 60 \\
\hline H31 & 4 & 4 & 3 & 7 & 5 & 11 & 10 & 5 & 7 & 56 \\
\hline $\mathrm{H} 32$ & 5 & 3 & 9 & 5 & 4 & 4 & 7 & 3 & 4 & 44 \\
\hline H33 & 3 & 2 & 4 & 7 & 4 & 3 & 7 & 4 & 4 & 38 \\
\hline H34 & 10 & 12 & 6 & 19 & 11 & 12 & 15 & 5 & 11 & 101 \\
\hline H35 & 7 & 6 & 5 & 12 & 9 & 8 & 10 & 5 & 5 & 67 \\
\hline $\mathrm{H} 36$ & 6 & 7 & 1 & 12 & 10 & 8 & 10 & 4 & 8 & 66 \\
\hline $\mathrm{H} 37$ & 3 & 3 & 8 & 8 & 6 & 6 & 8 & 3 & 5 & 50 \\
\hline H38 & 1 & 1 & 8 & 2 & 2 & 4 & 6 & 1 & 2 & 27 \\
\hline $\mathrm{H} 39$ & 9 & 11 & 2 & 18 & 10 & 11 & 15 & 5 & 11 & 92 \\
\hline $\mathrm{H} 40$ & 9 & 11 & 0 & 18 & 11 & 11 & 16 & 4 & 11 & 91 \\
\hline $\mathrm{H} 41$ & 3 & 5 & 0 & 3 & 5 & 0 & 0 & 1 & 2 & 19 \\
\hline $\mathrm{H} 42$ & 1 & 1 & 0 & 0 & 1 & 0 & 0 & 1 & 0 & 4 \\
\hline $\mathrm{H} 43$ & 0 & 0 & 1 & 0 & 2 & 0 & 0 & 1 & 1 & 5 \\
\hline $\mathrm{H} 44$ & 0 & 0 & 0 & 0 & 2 & 0 & 0 & 1 & 1 & 4 \\
\hline $\mathrm{H} 45$ & 2 & 0 & 4 & 1 & 0 & 0 & 0 & 0 & 0 & 7 \\
\hline $\mathrm{H} 46$ & 6 & 5 & 4 & 7 & 7 & 3 & 4 & 1 & 3 & 40 \\
\hline $\mathrm{H} 47$ & 0 & 0 & 0 & 0 & 0 & 0 & 0 & 0 & 0 & 0 \\
\hline $\mathrm{H} 48$ & 1 & 1 & 1 & 1 & 1 & 1 & 2 & 0 & 1 & 9 \\
\hline $\mathrm{H} 49$ & 0 & 0 & 0 & 0 & 0 & 0 & 0 & 0 & 0 & 0 \\
\hline $\mathrm{H} 50$ & 0 & 0 & 0 & 0 & 0 & 0 & 0 & 0 & 0 & 0 \\
\hline $\mathrm{H} 51$ & 0 & 0 & 0 & 0 & 0 & 0 & 0 & 0 & 0 & 0 \\
\hline H52 & 3 & 2 & 1 & 2 & 4 & 0 & 1 & 1 & 1 & 15 \\
\hline H53 & 1 & 1 & 1 & 1 & 1 & 1 & 1 & 0 & 2 & 9 \\
\hline H54 & 1 & 2 & 0 & 0 & 1 & 0 & 0 & 1 & 0 & 5 \\
\hline
\end{tabular}


28 a 30 de SETEMBRO

\begin{tabular}{|c|c|c|c|c|c|c|c|c|c|c|}
\hline $\mathrm{H} 55$ & 1 & 1 & 0 & 0 & 1 & 0 & 0 & 0 & 0 & 3 \\
\hline $\mathrm{H} 56$ & 0 & 0 & 0 & 0 & 0 & 0 & 0 & 1 & 1 & 2 \\
\hline $\mathrm{H} 57$ & 3 & 2 & 2 & 2 & 4 & 1 & 1 & 2 & 2 & 19 \\
\hline $\mathrm{H} 58$ & 7 & 11 & 9 & 19 & 10 & 12 & 16 & 5 & 11 & 100 \\
\hline $\mathrm{H} 59$ & 0 & 0 & 0 & 0 & 0 & 0 & 0 & 0 & 0 & 0 \\
\hline $\mathrm{TH}^{*}$ & 43 & 41 & 37 & 41 & 44 & 38 & 35 & 43 & 44 & - \\
\hline
\end{tabular}

* TH é o total de habilidades distintas trabalhadas em um ano do período de estudo.

Fonte: Os autores

O primeiro resultado a ser destacado no Quadro 4 é que $85 \%(50)$ das 59 habilidades foram exercitadas ao menos uma vez pelas atividades do grupo no período estudado. As 10 habilidades mais exercitadas ( 100 ou mais vezes) correspondem a $17 \%$ do total e sendo 9 do tipo softskill (H16, H17, H21, H23, H25, H27, H28, H29 e H34) e uma habilidade técnica (H58). As 9 habilidades não técnicas se relacionam à comunicação, à autonomia, ao pensamento crítico, ao aprendizado contínuo e à ética profissional, consequência direta da natureza do programa de educação tutorial marcada por trabalho em grupo cooperativo, estrutura horizontal e busca por consenso. Além disso $25 \%$ das 59 habilidades foram exercitadas mais de 50 vezes nos nove anos do estudo. $O$ grupo de atividades que foram exercitadas mais de 10 vezes ao longo dos nove anos correspondem a $22 \%$ do total das 59 habilidades.

Quanto às 9 habilidades que não foram trabalhadas durante o período de estudo, 8 $(\mathrm{H} 8, \mathrm{H} 10, \mathrm{H} 12, \mathrm{H} 13, \mathrm{H} 47, \mathrm{H} 49, \mathrm{H} 50 \mathrm{e} \mathrm{H} 51)$ referem-se às habilidades técnicas ligadas à tecnologias e equipamentos de custo relativamente alto. A ausência do desenvolvimento dessas habilidades nas atividades do PET Engenharia Civil, se explicam pela dificuldade de acesso no contexto da universidade pública em que se insere o grupo.

Ao longo dos anos observa-se, da última linha do Quadro 4, uma regularidade da diversidade de atividades desempenhadas pelo grupo, uma vez que em média $70 \%$ (41) das 59 Habilidades são exercitadas ao menos uma vez por ano. Esse resultado é importante para o desenvolvimento dos indivíduos do grupo uma vez que a permanência dos discentes no grupo tem sido em média de 1,46 ano nos últimos 6 anos.

No Quadro 5 apresentam-se as quantidades de habilidades distintas envolvidas na execução de uma determinada habilidade Ai. As linhas do Quadro 5 representam um dos 51 tipos de atividades listadas no Quadro 3, enquanto as colunas representam os anos do período 2013-2021. As células preenchidas com o símbolo "- "indicam que um determinado tipo de atividade Ai não ocorreu no determinado ano. A eventual variação do número de habilidades distintas para um mesmo tipo de atividade ao longo dos anos é reflexo de eventuais modificações na metodologia dessas atividades conforme indicação dos relatórios e planejamentos consultados.

Quadro 5 - Quantidade de habilidades distintas por atividades nos anos 2013-2021.

\begin{tabular}{|c|c|c|c|c|c|c|c|c|c|}
\hline A/Ano & 2013 & 2014 & 2015 & 2016 & 2017 & 2018 & 2019 & 2020 & 2021 \\
\hline A1 & - & - & - & - & - & - & - & - & 20 \\
\hline A2 & - & - & - & - & - & - & 31 & - & - \\
\hline A3 & 31 & 31 & 31 & 31 & 31 & 31 & 31 & 31 & 31 \\
\hline A4 & - & - & - & - & - & - & 25 & - & - \\
\hline A5 & - & - & - & 16 & - & - & - & - & - \\
\hline A6 & - & - & - & - & - & - & - & 26 & 26 \\
\hline A7 & - & 19 & 19 & - & - & - & - & - & - \\
\hline A8 & 20 & - & - & - & - & - & - & - & - \\
\hline A9 & - & - & 22 & - & - & - & - & - & - \\
\hline A10 & 27 & 27 & 27 & 27 & 27 & - & 27 & - & - \\
\hline A11 & 31 & 31 & 31 & 31 & 31 & 31 & 31 & 31 & 31 \\
\hline A12 & - & - & - & - & - & - & - & - & 19 \\
\hline
\end{tabular}


28 a 30 de SETEMBRO

\begin{tabular}{|c|c|c|c|c|c|c|c|c|c|}
\hline A13 & - & - & - & 27 & 27 & - & - & - & - \\
\hline A14 & 22 & 22 & - & - & - & - & - & - & - \\
\hline A15 & - & - & - & - & - & 21 & - & - & - \\
\hline A16 & - & - & - & - & - & - & 17 & 17 & 17 \\
\hline $\mathrm{A} 17$ & - & 17 & - & - & - & - & - & - & - \\
\hline A18 & 22 & 22 & 22 & 22 & 22 & 22 & 22 & - & - \\
\hline A19 & - & - & - & 23 & - & - & - & - & - \\
\hline A20 & - & - & - & 14 & - & - & - & - & - \\
\hline A21 & - & - & - & 17 & - & - & - & - & - \\
\hline A22 & 27 & 27 & - & - & 27 & - & - & - & - \\
\hline A23 & 22 & - & - & - & - & - & - & - & - \\
\hline A24 & - & - & - & - & - & 25 & 25 & - & - \\
\hline A25 & - & - & - & - & 22 & 22 & 22 & - & - \\
\hline A26 & - & - & - & - & 20 & - & - & - & - \\
\hline A27 & - & - & - & 31 & - & 31 & 31 & - & - \\
\hline A28 & 34 & - & - & - & - & - & - & - & - \\
\hline A29 & - & - & 28 & - & - & - & - & - & - \\
\hline A30 & - & - & 26 & 26 & - & - & - & - & - \\
\hline A31 & - & - & - & - & - & - & - & - & 32 \\
\hline A32 & - & - & - & 26 & - & - & - & - & - \\
\hline A33 & - & - & - & 17 & - & - & - & - & - \\
\hline A34 & - & - & - & 18 & - & - & - & - & - \\
\hline A35 & - & - & - & - & 30 & - & - & - & - \\
\hline A36 & 26 & - & - & - & - & 26 & 26 & 26 & 26 \\
\hline A37 & - & - & - & - & - & 24 & - & - & - \\
\hline A38 & 20 & 20 & - & - & 20 & - & - & - & - \\
\hline A39 & - & - & - & - & - & - & 17 & - & - \\
\hline A40 & - & - & - & - & - & - & 21 & - & - \\
\hline A41 & - & - & - & - & - & - & - & - & 23 \\
\hline A42 & - & 14 & - & - & - & - & - & - & - \\
\hline A43 & - & - & - & 24 & - & - & - & - & - \\
\hline A44 & - & 18 & 18 & 18 & 18 & 18 & 18 & 18 & 18 \\
\hline A45 & - & 18 & - & - & - & - & - & - & - \\
\hline A46 & - & - & - & 13 & - & - & - & - & - \\
\hline A47 & - & - & - & - & - & - & - & 38 & 38 \\
\hline A48 & 24 & 21 & 21 & 21 & 31 & 21 & 23 & 23 & 23 \\
\hline A49 & - & - & - & 17 & - & - & - & - & - \\
\hline A50 & - & - & - & - & - & 29 & 29 & - & - \\
\hline A51 & - & - & - & - & - & - & 18 & & - \\
\hline
\end{tabular}

Fonte: Os autores

A análise dos resultados do Quadro 5 permitem a avaliação da eficiência dos tipos de atividades realizadas pelo grupo PET Engenharia Civil da UFPR no exercício das habilidades dos Quadros 1 e 2. Uma análise global mostra que em média cada atividade desempenhada pelo grupo provê aos seus participantes oportunidades de desenvolvimento de 24 habilidades distintas, o que constitui $41 \%$ do total de habilidades do estudo de Akyazi e coautores (2020). A atividade A47 - Telhado Verde - foi a atividade que, no período estudado, apresentou a maior diversidade de habilidades exercitadas - 38 (64\% do total). Trata-se de uma atividade baseada na solução de um problema técnico, envolvendo pesquisa, ensino e extensão, outras atividades semelhantes como as A31 e A35 também de caráter similar apresentam diversidade de habilidades trabalhadas. Outras 3 atividades com maior diversidade de habilidades têm objetivos não técnicos que, contudo ao envolver organização de eventos, ministração de cursos e as atividades de organização e estrutura interna do grupo fornecem oportunidades de exercício de muitas dos softskills listados nos Quadros 1 e 2. 


\section{CONCLUSÕES}

O estudo realizado permitiu a avaliação das atividades desenvolvidas pelo grupo PET Engenharia Civil quanto à formação profissional de engenheiros civis segundo uma lista de 59 habilidades vistas como tendência em um futuro próximo quanto à relevância no cenário de modificação do mercado de trabalho da indústria da construção civil.

Ao longo de 9 anos (2013-2021) as atividades planejadas pelo grupo PET Engenharia Civil forneceram aos seus integrantes oportunidades de desenvolvimento em $85 \%$ das 59 habilidades anteriormente apontadas. Tal resultado demonstra a eficiência e a característica de formação profissional ampla do programa de educação tutorial na área de Engenharia Civil. As habilidades com o maior número de oportunidades de desenvolvimento oferecidas pelas atividades trabalhadas estão relacionadas ao trabalho em grupo, como por exemplo a questão de gerenciamento de projetos, da comunicação, da autonomia, da iniciativa, do aprendizado contínuo, das relações interpessoais, da resolução e do gerenciamento de problemas e conflitos. Tal fato demonstra a eficiência no desenvolvimento profissional que o caráter do programa de educação tutorial marcado pela horizontalidade, trabalho colaborativo e busca por consenso.

Uma vez que em média $70 \%$ das 59 habilidades são contempladas ao menos uma vez pelas atividades anualmente desempenhadas pelo grupo, pode-se dizer que 0 planejamento do grupo tem sido realizado de modo eficiente, uma vez que os recursos financeiros (bolsa e custeio) e a carga horária dos integrantes tem sido empregados para uma formação ampla e condizente com as futuras necessidades do mercado e da sociedade.

Tanto atividades organizacionais (interna ou de eventos científicos, técnicos e de extensão) quanto atividades de caráter de resolução de problemas práticos de engenharia (articulando ao menos duas das frentes da tríade ensino-pesquisa-extensão) se mostram como as atividades mais eficientes no desenvolvimento das habilidades profissionais consideradas.

Grande parte das habilidades não contempladas pelas atividades do grupo no período de nove anos deste estudo referem-se à habilidades técnicas ligadas a tecnologias e equipamentos de custo relativamente alto, cujo acesso por parte do grupo, inserido no contexto do ensino público superior, tem sido limitado, uma vez que o regramento da verba de custeio do programa impede o seu uso em material permanente.

Este estudo poderá servir como base para futuros planejamentos do grupo, pois como a análise é realizada tendo por base as habilidades e as atividades desenvolvidas pelo grupo, este levantamento proporciona uma ferramenta para escolha de atividades eficientes no desenvolvimento das habilidades necessárias aos futuros engenheiros civis.

\section{Agradecimentos}

Os autores agradecem ao FNDE/MEC pela concessão das bolsas do Programa de Educação Tutorial do PET e à infraestrutura concedida pelo CESEC - Centro de Estudos em Engenharia Civil e ao Departamento de Construção Civil da UFPR.

\section{REFERÊNCIAS}

AKYAZI, Tugce; ALVAREZ, Irantzu; ALBERDI, Elisabete; OYARBIDE-ZUBILLAGA, Aitor; GOTI, Aitor; BAYON, Felix. Skills needs of the civil engineering sector in the european union countries: Current situation and future trends. Applied Sciences (Switzerland), [S. 
I.], v. 10, n. 20, p. 1-24, 2020. DOI: 10.3390/app10207226.

APARICIO, Angel C. et al. Tradition and Innovation in Teaching. Journal of Professional Issues in Engineering Education and Practice, [S. I.], v. 133, n. 4, p. 340-349, 2007. DOI: 10.1061/(ASCE)1052-3928(2007)133.

CAMPUS, Kingswood; PENRITH, S. Engineering education - is problem-based or projectbased learning the answer? Australasian Journal of Engineering Education, [S. I.], v. 3, p. ISSN 1324-5821, 2003. Disponível em:

http://www.aaee.com.au/journal/2003/mills_treagust03.pdf.

FELDER, Richard M.; BRENT, Rebecca. The intellectual development of science and engineering students. Part 2: Teaching to promote growth. Journal of Engineering Education, [S. I.], v. 93, n. 4, p. 279-291, 2004. a. DOI: 10.1002/j.21689830.2004.tb00817.x.

FELDER, Richard M.; BRENT, Rebecca. The intellectual development of science and engineering students. Part 1: Models and challenges. Journal of Engineering Education, [S. I.], v. 93, n. 4, p. 269-277, 2004. b. DOI: 10.1002/j.2168-9830.2004.tb00816.x.

MARTIN, Maria da Graça Moraes Braga. O Programa De Educação Tutorial-Pet : Formação Ampla Na Graduação. [S. I.], 2005.

NAVON, Ronie. The Last Lecture: The Need for Civil Engineering Education to Stimulate Thinking Instead of Only Teaching. Journal of Construction Engineering and Management, [S. I.], v. 146, n. 5, p. 02520001, 2020. DOI: 10.1061/(asce)co.19437862.0001805 .

PESQUISA; SILVA, J. A. C.; PRADO, C. R.; GONÇALVES, L. W.; SIMIONATO, N. M.; OLIVEIRA, N. C.; GAVASSONI, E. BIM: UMA ANÁLISE DA GRADE CURRICULAR DO CURSO DE ENGENHARIA CIVIL DA UFPR. [S. I.], 2019.

PRIMO, Murilo Ferreira; DIAS, Gabriel Angelo Juc; SILVA, João Antonio Comine da;; NETO, Elvidio Gavassoni. INSTITUCIONALIZACCÃO DAS ATIVIDADES DO PET COMO FORMA DE RESISTÊNCIA E CONSOLIDAÇÃO DO PROGRAMA. [S. I.], 2019.

SCHWAB, Klaus. A Quarta Revolução Industrial. [s.I: s.n.].

SILVA, Marcio Roque Dos Santos Da; OLAVE, Maria Elena Leon. Contribuições Das Tecnologias Digitais Associadas À Indústria 4.0 Para a Formação Profissional. Revista Gestão e Desenvolvimento, [S. I.], v. 17, n. 2, p. 82, 2020. DOI:

10.25112/rgd.v17i2.2047.

TIBONI, Giovanna Reinehr; PEREIRA, Bernardinis Márcia de Andrade. INOVAÇÕES NO CURSO DE ENGENHARIA CIVIL DA UNIVERSIDADE FEDERAL DO PARANÁ (UFPR) SOB A ÓTICA DAS METODOLOGIAS ATIVAS. [S. I.], p. 1-21, 2019.

UFPR, PET Engenharia Civil. Planejamento Anual 2013. [S. I.], 2013. a.

UFPR, PET Engenharia Civil. Relatório de Atividades 2013. [S. I.], 2013. b. 
UFPR, PET Engenharia Civil. Planejamento Anual 2014. [S. I.], p. 1-10, 2014. a.

UFPR, PET Engenharia Civil. Relatório de Atividades 2014. [S. I.], 2014. b.

UFPR, PET Engenharia Civil. Planejamento Anual 2015. [S. I.], 2015. a.

UFPR, PET Engenharia Civil. Relatório de Atividades 2015. [S. I.], 2015. b.

UFPR, PET Engenharia Civil. Planejamento Anual 2016. [S. I.], 2016. a.

UFPR, PET Engenharia Civil. Relatório de Atividades 2016. [S. I.], 2016. b.

UFPR, PET Engenharia Civil. Planejamento Anual 2017. [S. I.], 2017. a.

UFPR, PET Engenharia Civil. Relatório de Atividades 2017. [S. I.], 2017. b.

UFPR, PET Engenharia Civil. Planejamento Anual 2018. [S. I.], 2018. a.

UFPR, PET Engenharia Civil. Relatório de Atividades 2018. [S. I.], 2018. b.

UFPR, PET Engenharia Civil. Planejamento Anual 2019. [S. I.], 2019. a.

UFPR, PET Engenharia Civil. Relatório de Atividades 2019. [S. I.], 2019. b.

UFPR, PET Engenharia Civil. Planejamento Anual 2020. [S. I.], 2020. a.

UFPR, PET Engenharia Civil. Relatório de Atividades 2020. [S. I.], 2020. b.

UFPR, PET Engenharia Civil. Planejamento Anual 2021. [S. I.], 2021.

VASCO, CELINE; FERREIRA, DEMIS TEILOR; BRANDALIZE, DIOGO CAVASSIN; PASQUAL, GUILHERME GARCIA; AGUIAR, GUILHERME VELOSO DE; BONILHA, HÉLDER HENRIQUE; DALMAGRO, IGOR; OLIVEIRA, LARISSA F. G. DE; PELISSARI, LUCAS BARBOSA; DIAS, MÁRCIO SILVESTRE; HECKE, MILDR, Ricardo. PET 25 ANOS. [S. I.], 2008.

\section{THE TUTORIAL EDUCATION PROGRAM AND THE TRAINING OF CIVIL ENGINEERS PREPARED FOR THE FUTURE LABOR MARKET}

Abstract: The fourth industrial revolution has brought to society the need to understand new technologies, improve non-technical skills and their applications in the job market. Civil engineers are part of this professional profile, as they work with the resolution of complex practical problems, their jobs require socio-behavioral skills and the use of new technologies aimed at productivity and environmental awareness. The Group of Tutorial Education Program (PET) Civil Engineering at the Federal University of Paraná is a pioneer education program with more than 37 years of history that seeks to integrate innovative actions articulating academic, research and extension society-oriented practices. The group PET develops his activities exercising collaborative work, horizontality and group work guided by a university professor. In order to analyze how much the PET group collaborates in the training of civil engineers, a study was carried out based on a list of 59 future professional 
skills required by European Union civil engineering job market. It was found that the group PET in the last 9 years has worked $85 \%$ of the afore mentioned skills. This results indicates that the PET program has a positive impact on the preparation of the workforce for the future.

Keywords: future skills, construction sector, Industry 4.0. 